\title{
PRIVAT -
}

\section{WIRTSCHAFTSLEHRE DER}

\section{UNTERNEHMUNG}

Von

\section{FRIEDRICH LEITNER}

PROFESSOR AN DER HANDELSHOCHSCHULE BERUN

ZWEITE UNVERANDERTE AUFLAGE

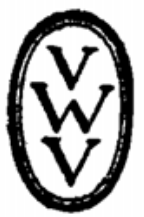

BERLIN UND LEIPZIG 1919

VEREINIGUNG WISSENSCHAFTLICHER VERLEGER

WALTER DE GRUYTER \& CO.

VORMALS G. J. GOSCHEN'SCHE VERLAGSHANDLUNG , J. GUTTENTAG, VERLAGSBUCHHANDLUNG , GEORG RBMER , KARL J. TROBNER , VET \& COMP. 
